alpha $(\mathrm{OH})$ vitamin D3 (alphacalcidiol) analogue was shown to induce less hypercalcemic side effects.

Objectives We organised open-label therapeutical trial in $20 \mathrm{RA}$ and 15 JRA patients who have had three months active disease on basic therapy. Therapy regime remained unchanged but alphacalcidiol in high oral dose was added during next three months $(2 \mu \mathrm{gr} /$ day in RA or $0,05 \mu \mathrm{gr} / \mathrm{kg} /$ day in JRA pts.).

Methods Spontaneous and PMA stimulated IL1 and TNF production was measured in all patients before and after the trial by ELISA method in PB mononuclear cells after $24 \mathrm{~h}$ cultures. GSH-Px (glutation-peroxidase) and SOD (superoxide-dismutase) activity was measured by RANDOX kits.

Results Three months high oral dose therapy significantly improved clinical signs of disease activity in correlation with parameters we evaluated. Increased IL1 and TNF production was significantly lowered after the trial and riched values in healthy controls. GSH-Px and SOD activity were lowered in all patients before entering into the stady. Alphacalcidiol therapy induced significant increase of GSH-Px and, on contrary, decrease of SOD activity. Positive correlation trends among this enzymes during disease activity were turned into negative according to disease improvement after the trial finished.

Conclusion High dose alphacalcidiol is powerful immunomodulatory agent as it is shown for other vitamin D3 analogues. Its main function seem to be stabilisation of impared immunoregulation in RA and JRA which could be of benefit as possible therapeutic agent.

\section{FRI0005 SAFETY OF NEW COX-2 INHIBITORS IN NSAID- INTOLERANT PATIENTS}

M Sánchez-Borges, A Capriles-Hulett, F Caballero, C Pérez. Allergy-Immunology, Clínica "El Avila", Caracas, Venezuela

\subsection{6/annrheumdis-2001.1134}

Background Non-steroidal anti-inflammatory drug (NSAID)-triggered urticaria and angioedema occurrs in up to $3 \%$ of the population. The tolerance to new COX-2 selective inhibitors in intolerant patients has not been determined.

Objectives To study the clinical tolerance to Rofecoxib and Celecoxib in patients sensitive to conventional NSAIDs who develop urticaria and/or angioedema when exposed to these drugs.

Methods Sixty three subjects (female 44, male 19, mean age $33.0 \pm 16.7$ years, range $11-74$ years) with challenge-proved urticaria/angioedema were studied. These patients were challenged by means of the single-blinded oral method, controlled with placebo.

Results Positive challenges were observed in 4 out of 53 subjects challenged with Rofecoxib (7.5\%) and in 13 out of 47 individuals submitted to oral provocation with Celecoxib (27.6\%) $(\mathrm{p}=$ $0.01)$.

Conclusion We conclude that: 1) The clinical tolerance to selective COX-2 inhibitors in NSAID-sensitive patients with skin manifestations differs between new drugs, which may be due to different degrees of enzyme selectivity or to other, not yet determined reasons.2) COX-1 inhibition would be involved in the production of cutaneous adverse reactions to NSAIDs.3) Controlled oral challenges are indicated especially for the management of sensitive individuals with chronic inflammatory and/or painful diseases.
FRI0006 DOUBLE-BLIND CLINICAL, LABORATORY AND ULTRASOUND ASSESSMENT OF THE EFFICACY OF ANTIBODIES TO INTERFERON-G (ANTI-IFN-G) IN RHEUMATOID ARTHRITIS (RA)

GV Loukina, YA Sigidin, OV Pushkova, ES Mach, SV Skurkovich, BS Skurkovich. Clinical Pharmacology, Institute of Rheumatology of RAMS, Moscow, Russia

10.1136/annrheumdis-2001.1135

\section{Background}

Objectives To objectively assess the efficacy and tolerability of anti-IFN-g in treating patients with active RA.

Methods Thirty patients with active RA were randomly assigned to receive intramuscularly injections of anti-IFN-g, antibodies to tumour necrosis factor a (anti-TNF) or placebo for 5 consecutive days (10 patients on each treatment regimen). The patients were clinically assessed daily for 7 days, then weekly up to the 28-th day. Clinical, laboratory and ultrasound indices were used for the evaluation of the treatment efficacy.

Results Both anti-cytokines were equally effective and significantly superior to placebo. Twelve patients stopped treatment due to the lack of efficacy: 1 on anti-TNF, 2 on anti-IFN-g and 9 on placebo. According to the physician?s assessment improvement was achieved by the 7-th day on anti-TNF in 9 patients, on anti-IFN-g in 7, and on placebo in 2. At the 28-th day the corresponding figures were 8,8 , and 0 , respectively. By the 28 -th day each of the anti-cytokine groups showed significant improvement in 5 clinical measures including numbers of swollen and painful joints, pain and fatigue. The single patient in the placebo group who completed the trial had no positive changes in the disease activity. The thickness of the inflamed synovial membrane assessed by the ultrasound method decreased significantly only on anti-IFN-g (both by the 7 -th and by the 28 -th days).

Conclusion The results obtained suggest that IFN-g plays an important role in the pathogenesis of RA. The use of antibodies to this cytokine can be considered as a promising approach to the therapy of RA, especially its treatment-resistant forms.

\section{FRI0007 PREFERENCES OF RHEUMATOLOGISTS FOR THE TREATMENT OF RHEUMATOID ARTHRITIS FLARE: IS THERE A STANDARD OF CARE?}

Y Yazici, D Erkan, MJ Harrison, SA Paget. Rheumatology, Hospital for Special Surgery, New York, USA

\subsection{6/annrheumdis-2001.1136}

Background Differences in physician evaluations, heterogeneity of disease and within patient variability contribute to the lack of a uniform definition of RA flare. As a result, a standardised approach to treatment of RA flare is not likely to exist. However, when presented with the same case scenario, rheumatologists' choice of treatment should establish a standard of care.

Objectives To identify physician medication preferences and the role of steroids in the treatment of rheumatoid arthritis (RA) flare.

Methods A questionnaire describing two versions of the same case of a previously stable RA patient maintained on $17.5 \mathrm{mg} /$ week of methotrexate (MTX), hydroxycholoroquine and NSAIDs, and presenting with signs and symptoms suggesting an increase in disease activity was developed; the second version included the addition of a standing dose of $5 \mathrm{mg}$ of prednisone. Rheumatologists were asked to identify their choice (s) of firstline therapy for each version of the case. The questionnaire was 